opment, Main Library, University of Saskatchewan, Saskatoon, Saskatchewan, S7N 0W0, Canada; Dennis Kimmage, Senior Assistant Librarian, Feinberg Library, State University College, Plattsburgh, NY 12901.

\section{UNIVERSITy LibraRIES SECTION (ULS)}

Vice-Chair/Chair-elect: Cynthia B. Duncan, Dean of Library Services, Old Dominion University, Norfolk, VA 23508; Melvin R. George, University Librarian and Director Learning Services, Northeastern Illinois University, Chicago, IL 60625.

Secretary (three-year term): Janice Koyama, Head Librarian, Moffitt Undergraduate Library, University of California, Berkeley, CA 94720; Ann K. Randall, Assistant University Librarian, Brown University, Providence, RI 02912.
Western European Specialists Section (WESS)

Vice-Chair/Chair-elect: Ross Atkinson, $\mathrm{Hu}-$ manities Bibliographer, Collection Management Division, Northwestern University Library, Evanston, IL 60201; Cecily Johns, Director of Collections and Information Services, University of Cincinnati, Cincinnati, $\mathrm{OH} 45221$.

Secretary (one-year term): Jeffry Larson, $\mathrm{Hu}$ manities Bibliographer, Yale University Library, New Haven, CT 06520; Fred J. Gitner, Librarian, French Institute/Alliance Francaise Library, New York, NY 10022.

Member-at-Large (one-year term): Marjorie A. Benedict, Bibliographer, State University of New York at Albany, Albany, NY 12222; Laszlo Kovacs, Head, Humanities Library, Purdue University, West Lafayette, IN 47907.

\title{
Tentative Schedule of
}

ACRL Midwinter Meetings

ALA Midwinter Meeting

San Antonio, Texas

JANUARY 8-13, 1983

\section{ACRL BOARD OF DiRECTORS}

First Board Meeting: Sunday, January 9, 2:00-5:30 p.m.

Second Board Meeting: Wednesday, January 12, 9:30 a.m. $-12: 30$ p.m

\section{ACRL Ad Hoc Task Forces}

Academic Libraries and Higher Education: Saturday, January 8, 2:00-4:00 p.m.

ACRL/ALA: Saturday, January 8, 2:00-4:00 p.m.

Library Performance Measures: Saturday, January 8, 2:00-4:00 p.m.

Library Schools and Academic Libraries: Saturday, January 8, 2:00-4:00 p.m.

Library Statistics: Saturday, January 8, 2:00-4:00 p.m.

Membership Promotion: Saturday, January 8, 8:00 a.m. $-12: 30$ p.m.

Orientation of New Committee Members and Committee Chairs: Saturday, January 8, 2:00-4:00 p.m.

Research Needs of Academic/Research Libraries: Saturday, January 8, 2:00-4:00 p.m.

\section{ACRL Divisional Committees}

Academic and Research Library Personnel Study Group: Monday, January 10, 8:00-11:00 a.m.

Academic or Research Librarian of the Year Award: Sunday, January 9, 8:00-9:00 a.m.;
Monday, January 10, 9:30 a.m.-12:30 p.m. *

Academic Status: Saturday, January 8, 8:00-10:00 p.m.; Wednesday, January 12, 8:00-11:00 a.m.

Appointments and Nominations: Saturday, January 8, 2:00-4:00 p.m.; Sunday, January 9, 9:30-11:00 a.m.*

Audiovisual: Sunday, January 9, 9:30-11:00 a.m.; Tuesday, January 11, 11:30 a.m.-12:30 p.m.

Books for College Libraries III: Saturday, January $8,5: 30-7: 00$ p.m.

Budget and Finance: Monday, January 10, 9:30 a.m.-12:30 p.m. and 2:00-5:30 p.m.; Tuesday, January 11, 9:30 a.m.-12:30 p.m. and 2:00-5:30 p.m.; Wednesday, January 12, 2:00-5:30 p.m.

College Library Standards: Sunday, January 9, 2:00-4:00 p.m.; Monday, January 10, 9:00-11:00 a.m.

Conference Program Planning CommitteeDallas, 1984: Monday, January 10, 8:00-9:00 a.m.

Conference Program Planning Committee-Los Angeles, 1983: Sunday, January 9, 11:30 a.m. $-12: 30$ p.m.

Constitution and Bylaws: Monday, January 10, 9:30-11:00 a.m.; Wednesday, January 12, 2:00-4:00 p.m.

Continuing Education: Sunday, January 9, 9:00-11:00 a.m.; Monday, January 10, 9:00-11:00 a.m.

Doctoral Dissertation Fellowship: Monday, Janu-

*Meetings with an asterisk are closed meetings. 
ary 10, 2:00-5:30 p.m.; Tuesday, January 11, 9:30 a.m. $-12: 00$ p.m.*

Legislation: Saturday, January 8, 2:00-4:00 p.m.; Monday, January 10, 2:00-5:30 p.m.

Membership: Saturday, January 8, 8:00 a.m. $-12: 30$ p.m.; Monday, January 10, 9:00-11:00 a.m.; Tuesday, January 11, 9:00-11:00 a.m.

Publications: Saturday, January 8, 2:00-4:00 p.m.; Monday, January 10, 2:00-4:00 p.m.

Samuel Lazerow Fellowship: Saturday, January 8, 2:00-5:30 p.m; Sunday, January 9, 9:30 a.m. $-12: 00$ p.m.*

Seattle National Conference Executive Committee: Monday, January 10, 9:30 a.m.-12:30 p.m.

Standards and Accreditation: Saturday, January 8 , 2:00-5:30 p.m.; Sunday, January 9, 9:00-11:00 a.m.

\section{ACRL COUNCILS}

Chapters: Sunday, January 9, 8:00-10:00 p.m.; Monday, January 10, 2:00-5:30 p.m.

\section{ACRL Editorial Boards}

Choice: Sunday, January 9, 9:30 a.m.-12:30 p.m.

College \& Research Libraries: Monday, January 10 , 2:00-4:00 p.m.

College \& Research Libraries News: Monday, January 10, 9:30-11:00 a.m.

Nonprint Media Publications: Monday, January 10, 11:30 a.m.-12:30 p.m.

Publications in Librarianship: Monday, January 10, 9:30 a.m. $-12: 30$ p.m.

\section{ACRL Discussion Groups}

Cinema Librarians: Sunday, January 9, 9:00-11:00 a.m.

English and American Literature: Monday, January 10, 2:00-4:00 p.m.

Extended Campus Library Services: Saturday, January 8, 2:00-4:00 p.m.; Monday, January 10, 2:00-4:00 p.m.

Fee-based Research in Academic Libraries: Sunday, January 9, 2:00-4:00 p.m.

Librarians of Library Science Collections: Monday, January 10, 9:00-11:00 a.m.

Personnel Administrators of Academic Research Libraries: Saturday, January 8, 4:30-5:30 p.m.; Sunday, January 9, 4:30-5:30 p.m.; Monday, January 10, 4:30-5:30 p.m.

Public Relations: Sunday, January 9, 2:00-4:00 p.m.; Monday, January 10, 2:00-4:00 p.m.

Staff Development in Academic Research Libraries: Saturday, January 8, 11:30 a.m.-12:30 p.m.; Sunday, January 9, 11:30 a.m.-12:30 p.m.; Monday, January 10, 11:30 a.m.-12:30 p.m.

Undergraduate Librarians: Monday, January 10, 2:00-4:00 p.m.

\section{ACRL SECTIONS}

Anthropology and Sociology Section
Executive Committee: Sunday, January 9, 11:30 a.m. $-12: 30$ p.m.

Conference Program Planning-Dallas, 1984: Sunday, January 9, 8:00-9:00 a.m.

Nominating: Sunday, January 9, 8:00-9:00 a.m.*

Review and Planning Committee: Sunday, January $9,9: 30-11: 00$ a.m.

Art Section

Executive Committee: Monday, January 10, 2:00-4:00 p.m.

Asian and African Section

Executive Committee: Sunday, January 9, 9:30-11:00 a.m.

Bibliographic Instruction Section

Executive Committee: Saturday, January 8, 11:30 a.m. $-12: 30$ p.m.

Executive Council: Sunday, January 9, 9:30-11:30 a.m.

Automation: Saturday, January 8, 2:00-4:00 p.m.

Clearinghouse: Saturday, January 8, 2:00-4:00 p.m.

Conference Program Planning-Dallas, 1984: Sunday, January 9, 2:00-4:00 p.m.; Monday, January 10, 2:00-4:00 p.m.

Conference Program Planning-Los Angeles, 1983: Saturday, January 8, 2:00-4:00 p.m.

Continuing Education: Saturday, January 8, 2:00-4:00 p.m.

Cooperation: Saturday, January 8, 2:00-4:00 p.m.

Education for Bibliographic Instruction: Monday, January 10, 8:30-11:00 a.m.

Machine-Assisted Bibliographic Instruction: Saturday, January 8, 2:00-4:00 p.m.; Monday, January 10, 9:30-11:00 a.m.

Nominating, 1983: Sunday, January 9, 2:00-4:00 p.m.*

Nominating, 1984: Saturday, January 8, 2:00-4:00 p.m.*

Orientation: Saturday, January 8, 8:00-11:00 a.m.

Policy and Planning: Sunday, January 9, 8:00-9:00 a.m.

Postconference Program Planning, 1983: Saturday, January 8, 2:00-4:00 p.m.

Research: Sunday, January 9, 8:00-9:00 a.m.

Research Evaluation: Saturday, January 8, 2:00-4:00 p.m.

College Libraries Section

Executive Committee: Sunday, January 9,

\section{You're Invited}

The $C \triangleleft R L$ News Editorial Board will have an informal meeting at ALA Midwinter in San Antonio on Monday, January 10, from 9:30 to 11:00 a.m. Anyone who wishes to meet the editor or members of the board is invited to attend. Your questions and comments are invited. 
9:00-11:00 a.m.; Tuesday, January 11, 8:00-9:00 a.m.

CLIP Notes: Saturday, January 8, 9:30-11:00 a.m.

Continuing Education: Sunday, January 9, 2:00-4:00 p.m.; Monday, January 10, 2:00-4:00 p.m.

Impact of National Library Policy and National Library Developments on the College Library: Saturday, January 8, 4:30-5:30 p.m.; Monday, January 10, 2:00-4:00 p.m.

Nominating: Saturday, January $8,2: 00-4: 00$ p.m.; Sunday, January 9, 9:30-11:00 a.m. ${ }^{*}$

Nonprint Material and the College Library: Monday, January 10, 11:30 a.m.-12:30 p.m.

Program Planning: Monday, January 10, 9:30 a.m. $-12: 30$ p.m.

Community and Junior College Libraries Section

Executive Committee: Saturday, January 8, 11:30 a.m.-12:30 p.m.; Sunday, January 9 , 4:30-5:30 p.m.

Bibliography: Saturday, January 8, 9:30-11:00 a.m.

Communications: Sunday, January 9, 9:30-11:00 a.m.

Instruction and Use: Saturday, January 8, 2:00-4:00 p.m.

Membership: Sunday, January 9, 2:00-4:00 p.m.

Nominating: Sunday, January 9, 8:00-9:00 a.m. ${ }^{*}$

Planning and Procedures: Sunday, January 9, 11:30 a.m. $-12: 30$ p.m.

Services to Disadvantaged Students: Saturday, January 8, 8:00-9:00 a.m.

Education and Behavioral Sciences Section

Executive Committee: Sunday, January 9, 8:00-10:00 p.m.; Monday, January 10, 4:30-5:30 p.m.

Bibliographic Instruction for Educators: January 9, 8:00-11:00 a.m.

Curriculum Materials/Problems of Access and Control (joint meeting): Sunday, January 9 , 2:00-5:30 p.m.

Program Planning: Sunday, January 9, 8:00-9:00 a.m.

Psychology/Psychiatry Committee: Monday, January 10, 2:00-4:00 p.m.

Standards for Education Library Services: Saturday, January 8, 2:00-5:30 p.m.

Law and Political Science Section

Executive Committee: Sunday, January 9, 9:00-11:00 a.m.

Rare Book and Manuscript Section

Executive Committee: Sunday, January 9, 9:00-11:00 a.m.; Monday, January 10, 2:00-4:00 p.m.

Continuing Education: Monday, January 10, 11:30 a.m. $-12: 30$ p.m.

Granting Awards for Exhibition Catalogs: Tuesday, January 11, 2:00-4:00 p.m.

Guidelines for Transferring Materials to Special
Collections: Tuesday, January 11, 8:00-10:00 p.m.

Information Exchange: Saturday, January 8, 2:00-4:00 p.m.

Manuscripts Curators Survey: Saturday, January 8, 9:00-11:00 a.m.

Postconference Planning Committee-Los Angeles, 1983: Monday, January 10, 9:00-11:00 a.m.

Preconference Planning Committee-Austin, 1984: Saturday, January 8, 4:30-5:30 p.m.

Security: Tuesday, January 11, 9:30-11:00 a.m.

Standards: Saturday, January 8, 8:00-10:00 p.m.; Monday, January 10, 8:00-10:00 p.m.

Standards for Professional Ethics: Sunday, January 9, 8:00-10:00 p.m.; Wednesday, January 12, 9:00-11:00 a.m.

Science and Technology Section

Nominating: Sunday, January 9, 8:00 a.m.-12:00 p.m.*

Oberly Award for Bibliography in the Agricultural Sciences: Sunday, January 9, 9:30-11:00 a.m.*

Program Planning: Sunday, January 9, 2:00-5:30 p.m.

Slavic and East European Section

Nominating: Saturday, January 8, 4:30-5:30 p.m.*

University Libraries Section

Steering Committee: Sunday, January 9, 9:00-11:00 a.m.

Nominating: Monday, January 10, 8:00-9:00 a.m.*

\section{New ACRL Discussion Group}

Librarians who have coordination or management responsibility for public service units are invited to attend an organizational meeting of a Heads of Readers/Public Services Discussion Group at ALA Midwinter. The intent of the group is to explore common problems in the delivery of public services in academic libraries and to consider a variety of issues, e.g., evaluation of programs, levels of services, deployment of personnel, etc., from a manager's perspective.

The organizational meeting is tentatively scheduled for Monday, January 10, 9:30-11:00 a.m. To confirm time and place, please check your conference program. The meeting is sponsored by ACRL and if sufficient interest is expressed, a petition will be circulated requesting official recognition as an ACRL discussion group. Contact: Peter D. Haikalis, Assistant Director for Readers Services, J. Paul Leonard Library, San Francisco State University, 1630 Holloway Avenue, San Francisco, California 94132 . 
Western European Specialists Section

Executive Committee: Tuesday, January 12, 2:00-4:00 p.m.

Conference Planning Committee-Dallas, 1984: Sunday, January 9, 9:30 a.m.-12:30 p.m.

Conference Planning Committee-Los Angeles, 1983: Saturday, January 8, 4:30-5:30 p.m.

Discussion Group: Monday, January 10, 2:00-4:00 p.m.

Nominating: Monday, January 10, 11:30 a.m. $-12: 30$ p.m. ${ }^{*}$

Publications/Membership Survey: Saturday, January 8, 2:00-4:00 p.m.

Renaissance Bibliography: Monday, January 10, 11:30 a.m.-12:30 p.m.

Research and Planning: Sunday, January 9, 9:30-11:00 a.m.

University of Minnesota Symposium Planning Group: Sunday, January 9, 2:00-5:30 p.m.

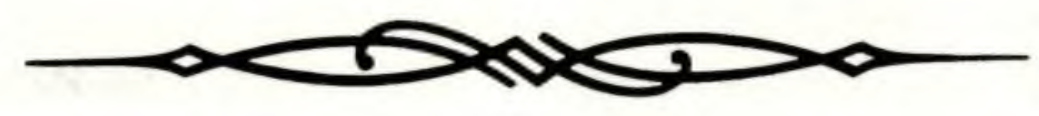

\section{LOOKING FOR}

- an organization dedicated to the Improvement of library service in academic and research settings?

- a means of establishing contacts?

- a vehicle for keeping abreast of the latest information in your fleld?

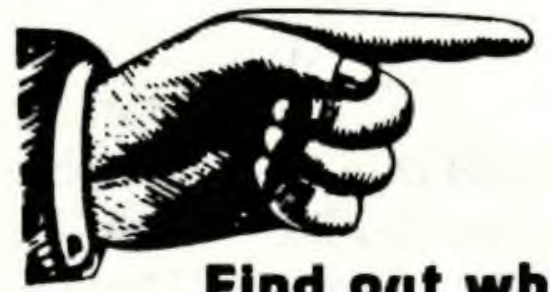

Then look to ACRL

Find out what we can do for you!

Please rush membership information to:

Name

Address

City State Zip

Return to:

ACRL/ALA

50 E. Haron St.

Chicago, II 60611

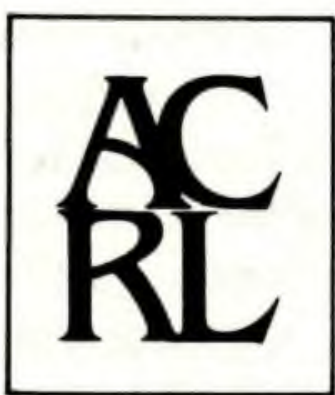

